\title{
Extended measurement of the proton spectrum with CALET on the International Space Station
}

\section{Kazuyoshi Kobayashi ${ }^{a, b, *}$ and Pier Simone Marrocchesi ${ }^{c, d}$ on behalf of the CALET Collaboration}

(a complete list of authors can be found at the end of the proceedings)

${ }^{a}$ Waseda Research Institute of Science and Engineering, Waseda University, 17 Kikuicho, Shinjuku, Tokyo 162-0044, Japan

${ }^{b} J E M$ Utilization Center, Human Spaceflight Technology Directorate, Japan Aerospace Exploration Agency, 2-1-1 Sengen, Tsukuba, Ibaraki 305-8505, Japan

${ }^{c}$ Dept. of Physical Sciences, Earth and Environment, Univ. of Siena, 53100 Siena, Italy

${ }^{d}$ INFN Sezione di Pisa, Polo Fibonacci, Largo B. Pontecorvo, 3-56127 Pisa, Italy

E-mail:kenkou@aoni.waseda.jp, marrocchesi@pi.infn.it

The Calorimetric Electron Telescope (CALET) is pursuing the direct measurement of the main components of high energy cosmic rays up to $\sim 1 \mathrm{PeV}$ in order to understand the cosmic ray acceleration and propagation. The detector consisting of a charge detector, an imaging calorimeter, and a total absorption calorimeter, is located on the International Space Station. The thickness of the calorimeter corresponds to 30 radiation lengths and to $\sim 1.3$ proton interaction lengths. We have taken data for more than five years so far: data taking has started in October 2015 and continues stably without any serious troubles. We present the latest result of proton spectrum analysis in the energy region from $30 \mathrm{GeV}$ to $60 \mathrm{TeV}$ with a fiducial geometrical factor of $\sim 510$ $\mathrm{cm}^{2} \mathrm{sr}$ and proton energy resolution is 30-40\%. The residual background is less than $10 \%$ in the $E<10 \mathrm{TeV}$ region. Compared to our previous result published in Physical Review Letters in 2019, more than two years of additional data have been analyzed. Spectral hardening around $500 \mathrm{GeV}$ is confirmed with higher statistics. We report our preliminary observation of a proton spectral softening around $10 \mathrm{TeV}$.

$37^{\text {th }}$ International Cosmic Ray Conference (ICRC 2021)

July 12th - 23rd, 2021

Online - Berlin, Germany

\footnotetext{
${ }^{*}$ Presenter
} 


\section{Introduction}

Direct measurements of cosmic-ray nuclei up to the PeV energy scale provide insight into the general phenomenology of cosmic-ray acceleration and propagation in the Galaxy. A possible charge-dependent cutoff in the spectra is hypothesized to explain the all-nuclei spectrum. Recently, a spectral hardening has been observed for several nuclei around a few hundreds $\mathrm{GeV}$ per nucleon. In the case of proton, we reported the hardening with much higher statistics [1] than previous experiments [2] [3]. Many theoretical models have been proposed to account for the spectral hardening including the presence of different cosmic ray sources, acceleration mechanisms, effect of diffusion in the Galaxy, and their superposition. In addition, DAMPE recently reported the observation of a proton spectral softening in the energy region around $10 \mathrm{TeV}$ [4]. It is crucial to observe accurately both the spectral hardening and the softening in order to understand the detailed mechanisms of cosmic-ray acceleration and propagation in the Galaxy. In this paper, the proton data of CALET are presented with increased statistics and extended energy range with respect to [1]. They show a softening of the proton spectrum above $10 \mathrm{TeV}$ which can be fitted simultaneously with the hardening of the spectrum around $500 \mathrm{GeV}$.

\section{CALET detector}

The CALorimetric Electron Telescope (CALET) [5], a space-based instrument optimized for the measurement of the all-electron spectrum [6,7] and equipped with a fully active calorimeter, can also measure cosmic-ray nuclei including proton in the energy range up to $1 \mathrm{PeV}$. The thickness of the calorimeter corresponds to 30 radiation lengths and to 1.3 proton interaction lengths.

The CALET detector consists of a charge detector (CHD), a 3 radiation-length thick imaging calorimeter (IMC) and a 27 radiation-length thick total absorption calorimeter (TASC), with a field of view of $45^{\circ}$ from zenith. A fiducial geometrical factor of approximate $510 \mathrm{~cm}^{2} \mathrm{sr}$ for particles penetrating CHD top to TASC bottom, with $2 \mathrm{~cm}$ margins at the first and the last TASC layers (acceptance A), and corresponding to about $40 \%$ of the total acceptance [7], is used in this analysis. The CHD, which identifies the charge of the incident particle, is comprised of a pair of plastic scintillator hodoscopes arranged in two orthogonal layers. The IMC is a sampling calorimeter alternating thin layers of Tungsten absorber with layers of scintillating fibers readout individually, also providing an independent charge measurement via multiple $\mathrm{dE} / \mathrm{dx}$ samples. The TASC is a tightly packed lead-tungstate $\left(\mathrm{PbWO}_{4}\right)$ hodoscope, measuring the energy of showering particles in the detector. More than 6 orders of magnitude in the energy region are covered by four different gain ranges. Due to the wide dynamic range of CALET as a single instrument, we can study the detailed shape of the spectrum without large systematic uncertainties from different detectors. Detailed description of the apparatus is given in the Supplemental Material of Ref. [6].

The instrument was launched on August 19, 2015 and emplaced on the Japanese Experiment Module-Exposed Facility (JEM-EF) on the International Space Station (ISS) with an expected mission duration of five years. Now the mission has been extended and the expected duration is nine years (or more) in total. Figure 1 shows the ISS and a schematic view of the CALET detector. Scientific observations started on October 13, 2015 and the detector operation continues without any serious troubles so far. 

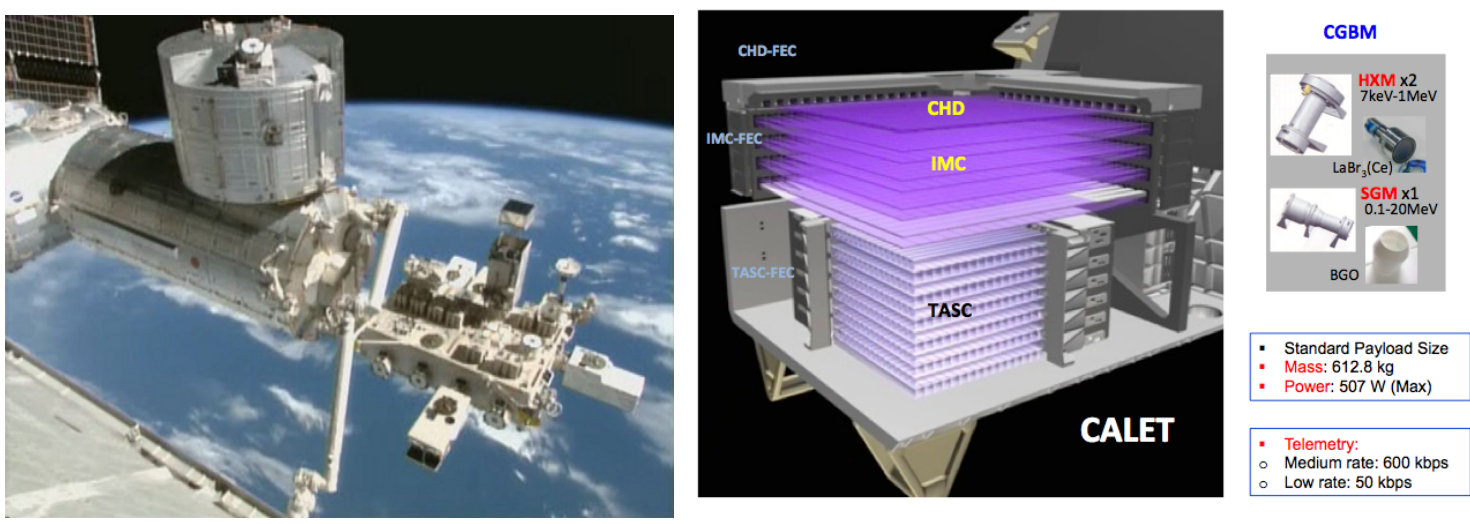

Figure 1: CALET detector. The left figure shows the ISS. ISS and the JEM-EF (bottom right corner of the picture) where CALET is located. The right figure shows a schematic view of the CALET detector.

\section{Data analysis}

We have analyzed the flight data collected for 1815 days from October 13, 2015 to September 30, 2020. The total observation livetime for the HE shower trigger [5] is 1539 days and the livetime fraction to total time is $84 \%$. In addition, the low-energy (LE) shower trigger operating at a high geomagnetic latitude [5] is used to extend the energy coverage toward the lower energy region. In spite of a limited livetime of 27.9 days, LE data provide sufficient statistics for protons below a few hundred GeV. Monte Carlo (MC) simulations reproducing the detailed detector configuration, physics processes, and detector signals, are based on the EPICS simulation package [8].

\subsection{Event selection and background}

In order to obtain the proton event sample, we apply the following selection criteria. We require that: (1) in the energy range, $E>300 \mathrm{GeV}$, the $\mathrm{HE}$ trigger should be asserted and the energy deposit sum of IMC 7th and 8th layers should be more than 50 minimum ionizing particles (MIPs) in either X and Y view. Also the energy deposit of TASC 1st layer should be more than $100 \mathrm{MIPs}$. In the energy range $E<300 \mathrm{GeV}$, the LE trigger should be asserted and the energy deposit sum of IMC 7th and 8th layers should be more than 5 MIPs in either X and Y view. Also the energy deposit of TASC 1st layer should be more than 10 MIPs. (2) Acceptance A is required as geometrical condition. (3) Kalman filter (KF) tracking in IMC should be adequate both in $\mathrm{X}$ and $\mathrm{Y}$ view. (4) Energy deposit inside one Moliere radius along the KF track in IMC should be less than $70 \%$ of total energy deposit. (5) Off-acceptance events are removed by the following two methods. One is that the maximum fractional energy deposit in a single TASC layer should be less than 0.4. The other is that the maximum energy deposit ratio of the edge channels to the maximum channels in each TASC layer should be less than 0.4. (6) Center of gravity of TASC energy deposit in $\mathrm{X} 1$ and $\mathrm{Y} 1$ layer should be consistent with the IMC track. (7) Shower start in IMC is required. (8) Charge is identified as proton using both CHD and IMC energy deposits. The Charge $(Z)$ is corrected for non-linear effects as $Z=a(E){\sqrt{N_{M I P}}}^{b(E)}$, where $N_{M I P}$ is the energy deposit in MIPs and $a(E)$ and $b(E)$ are energy dependent parameters determined using the MC simulations 
so that the average $Z$ should be 1 for proton and 2 for helium. The selection criteria are determined to keep the efficiency at the $95 \%$ level for the lower $Z$ side and $98 \%$ for the higher $Z$ side. Criterion (4) is required to remove electron background. Criterion (5) removes particles entering from the side of the detector. Criterion (6) remove mis-reconstructed events.

Background is estimated with MC simulations of cosmic ray protons, helium and electrons. After applying all the event selections, the dominant background comes from the off-acceptance protons below $\sim 5 \mathrm{TeV}$. Above, helium is the main background source.

\subsection{Energy unfolding}

Figure 2 shows a proton candidate with an energy deposit of $2.9 \mathrm{TeV}$ in the detector. The event example demonstrates our capability to reconstruct and identify high energy protons. Because of the limited energy resolution (30-40\%), energy unfolding is required to estimate the primary energy distribution. It is important, therefore, to infer the detector response at the highest energies covered by the analysis. First, we build a response matrix connecting true and observed energy spectra using MC simulation. Then, we apply an iterative unfolding procedure based on Bayes theorem taking into account helium and electron background consideration.
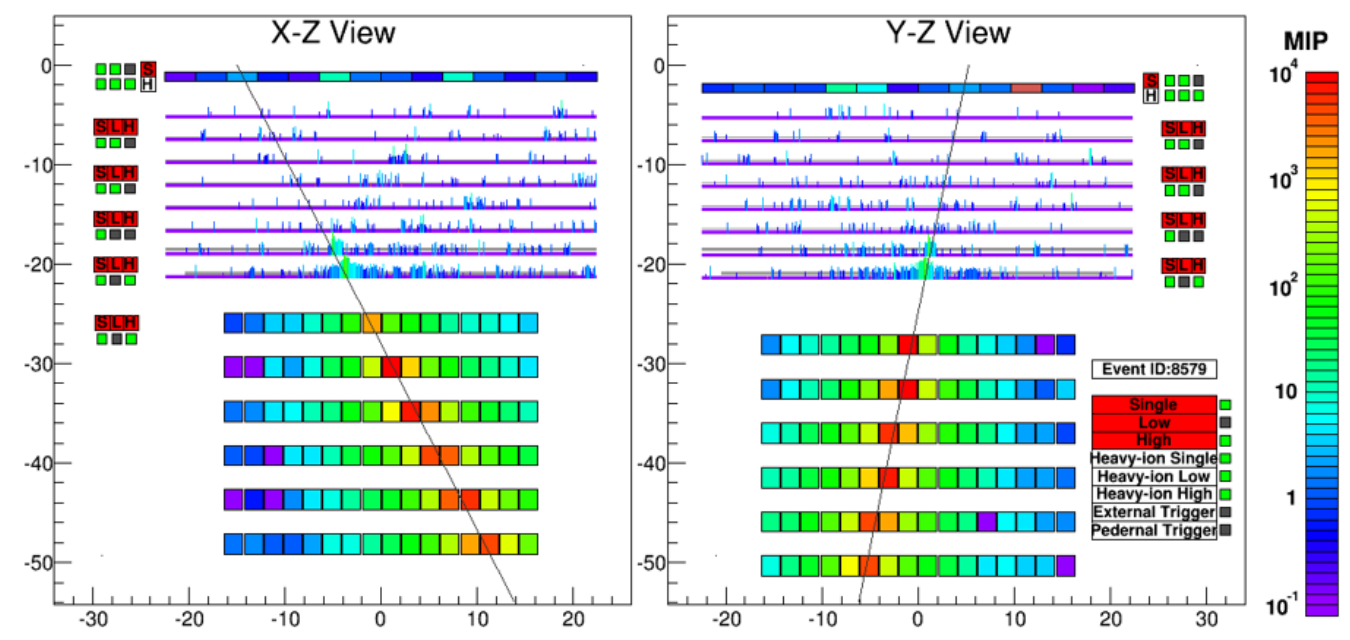

Figure 2: Proton event display with $2.9 \mathrm{TeV}$ energy deposit.

\subsection{Systematic uncertainty}

Two components are taken into account as systematic uncertainties. One is the energy independent component, which is $4.1 \%$ in total. It contains the uncertainties on livetime (3.4]\%), radiation environment (1.8\%), and long-term stability $(1.4 \%)$. The other one is the energy dependent component, which is less than $10 \%$ for $E<10 \mathrm{TeV}$. We take into account the uncertainties of MC model dependence, IMC track consistency with TASC, shower start in IMC, charge identification, energy unfolding, and beam test configuration. For $E>10 \mathrm{TeV}$, the uncertainties of MC model dependence and charge identification become dominant. In the interval $10<E<60 \mathrm{TeV}$ the uncertainty is $30 \%$ at maximum. Figure 3 shows the systematic uncertainty in the HE sample as a function of energy. 


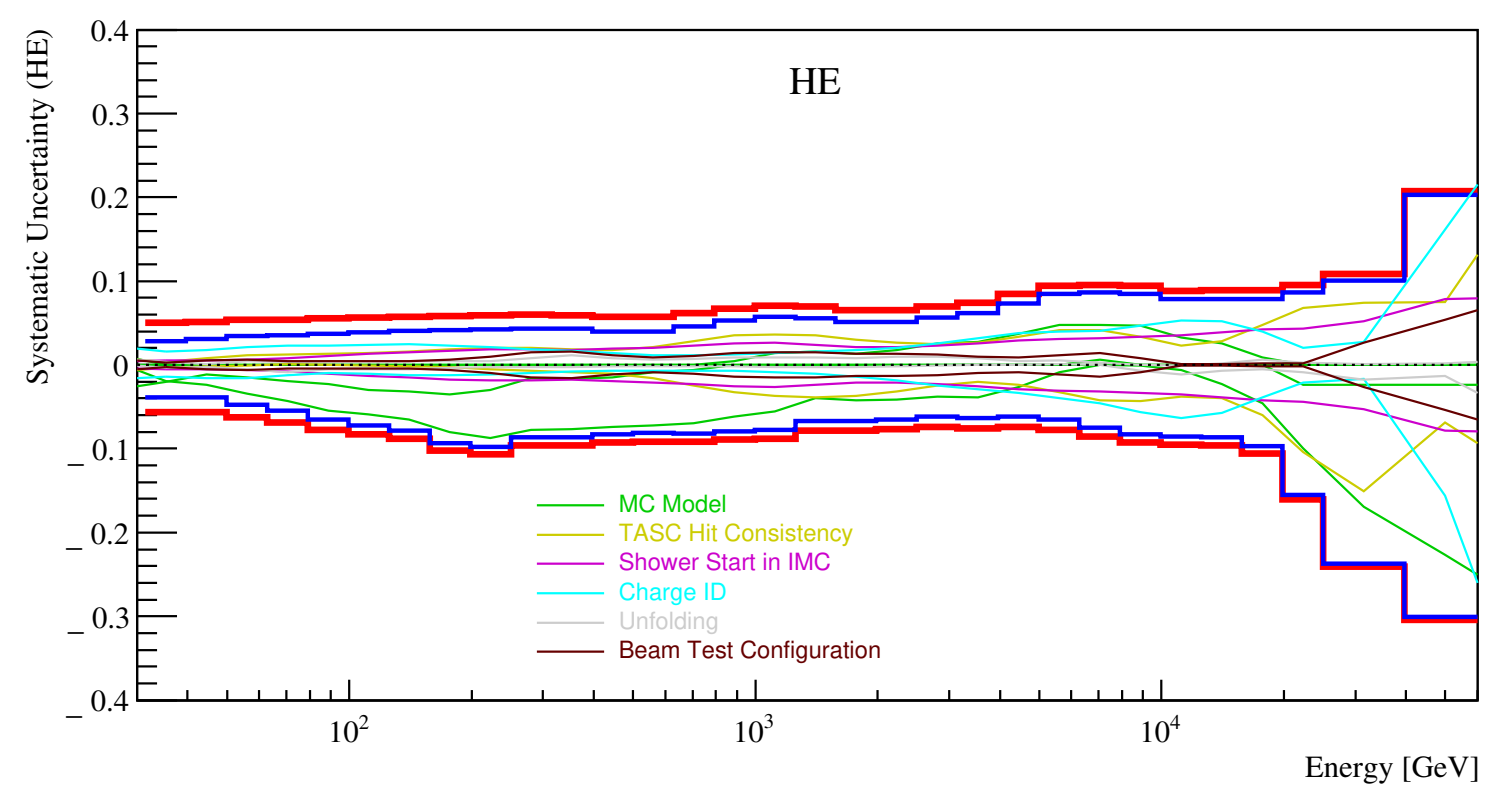

Figure 3: Systematic uncertainty in HE sample. The bold blue line shows the sum of the energy dependent systematic uncertainties. The bold red line shows the total systematic uncertainty including the energy independent uncertainties. The thin lines show the breakdown of the energy dependent uncertainty.

\subsection{Proton spectrum}

The proton spectrum $\Phi(E)$ is obtained from the following equation:

$$
\Phi(E)=\frac{N(E)}{S \Omega T \Delta E \epsilon(E)},
$$

where $N(E)$ is the number of selected events in $E=E+\Delta E, S \Omega$ is the geometrical acceptance $\left(510 \mathrm{~cm}^{2} \mathrm{sr}\right.$ ), $T$ is livetime (1539 days for HE and 27.9 days for LE, respectively), $\Delta E$ is the energy bin width, and $\epsilon(E)$ is the detection efficiency. Figure 4 shows the proton spectrum in the energy region from $30 \mathrm{GeV}$ to $60 \mathrm{TeV}$, compared with AMS-02, CREAM-III, and DAMPE. In the low energy region with $E<200 \mathrm{GeV}$, the result is fully consistent. In the higher energy region, a systematic difference is observed, but the difference is within the errors. We confirmed the presence of a spectral hardening around $500 \mathrm{GeV}$ in [1]. We also observe a spectral softening around 10 $\mathrm{TeV}$. We have tested two independent analyses with different efficiencies and the two results are consistent.

In order to calculate the behavior of the spectral hardening and softening quantitatively, we apply spectral fitting to the proton spectrum using a double broken power law function defined as follows:

$$
\Phi^{\prime}=E^{2.7} \times C \times\left(1-\frac{p_{0}}{E}-\frac{p_{1}}{E^{2}}\right) \times\left(\frac{E}{45}\right)^{\gamma} \times\left(1+\left(\frac{E}{E_{0}}\right)^{S}\right)^{\frac{\Delta \gamma}{S}} \times\left(1+\left(\frac{E}{E_{1}}\right)^{S}\right)^{\frac{\Delta \gamma_{1}}{S}}
$$




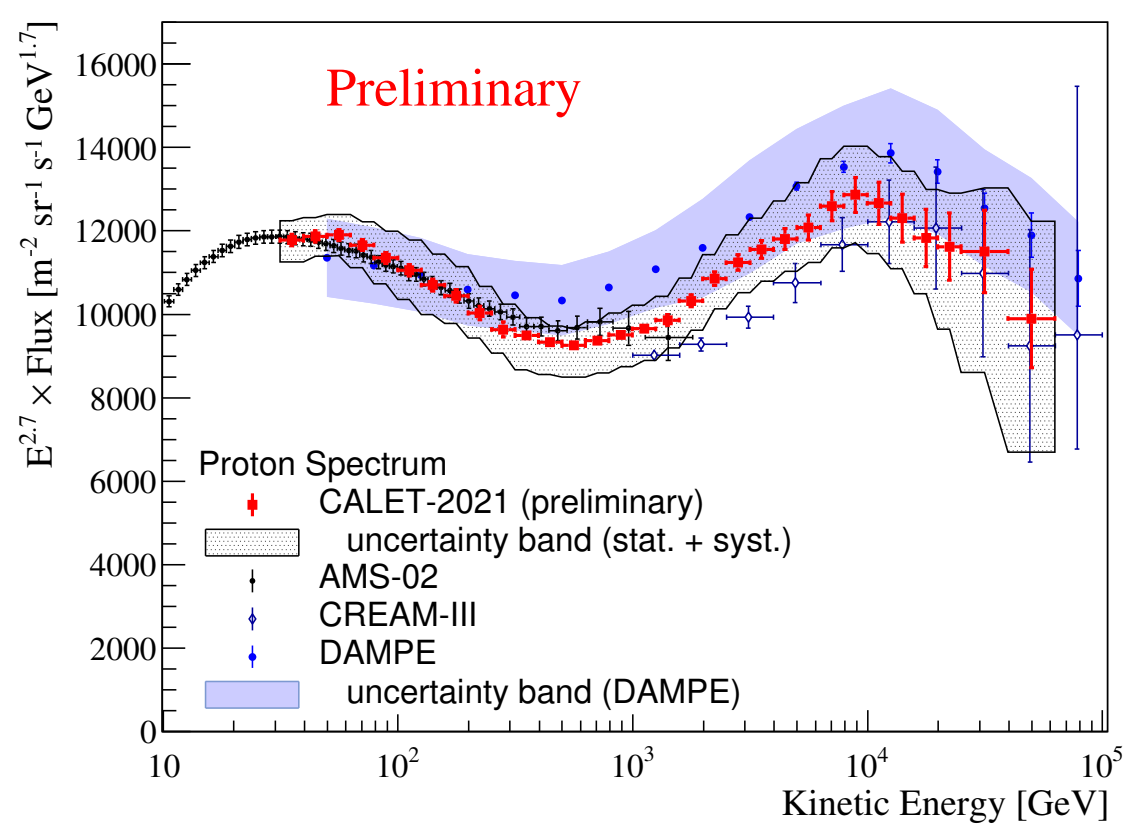

Figure 4: Proton spectrum measured by CALET (red circles) compared with the experimental results of AMS02 [2], CREAM-III [3], and DAMPE [4]. The hatched band shows the total uncertainty for CALET. The dark blue colored band shows the total uncertainty for DAMPE.

where $\Phi^{\prime}$ is the proton flux $\times E^{2.7}, C$ is the normalization factor, $p_{0}$ and $p_{1}$ are the fitting parameters for the low energy region, $S$ is the smoothness parameter, $\gamma$ is the spectral index, $\Delta \gamma$ is the spectral variation at hardening, $E_{0}$ is the hardening break energy, $\Delta \gamma_{1}$ is the spectral index variation at softening, and $E_{1}$ is the softening break energy.

In figure 5, the black filled circles show the data with statistical errors and the red line shows the best fitted function. The $\chi^{2}$ is 2.9 with 22 degrees of freedom. The best fitted parameters are: $p_{0}=9.1 \pm 26, p_{1}=-6.6 \pm 470, \gamma=-2.9 \pm 0.3, S=2.1 \pm 2.0, \Delta \gamma=(4.4 \pm 3.8) \times 10^{-1}$, $E_{0}=(5.5 \pm 1.3) \times 10^{2} \mathrm{GeV}, \Delta \gamma_{1}=(-4.4 \pm 3.0) \times 10^{-1}$, and $E_{1}=(1.1 \pm 0.4) \times 10^{4} \mathrm{GeV}$. The hardening starts at $550 \pm 130 \mathrm{GeV}$ and the softening starts at $11 \pm 4 \mathrm{TeV}$.

\subsection{Discussion}

The low energy part of the proton spectrum from $30 \mathrm{GeV}$ to $1 \mathrm{TeV}$ is consistent with AMS-02 and DAMPE. The high energy part of the spectrum from $1 \mathrm{TeV}$ to $60 \mathrm{TeV}$ is systematically lower than that of DAMPE, though the difference is within the uncertainties. On the other hand, the spectrum is higher than that of CREAM-III. The spectral hardening break energy observed in CALET $(550 \pm 130 \mathrm{GeV})$ is consistent within the errors with that of AMS- $02\left(336_{-44}^{+68}(\mathrm{fit})_{-28}^{+66}(\mathrm{sys}) \pm 1(\mathrm{sol}) \mathrm{GV}\right.$ [2]) and DAMPE (480 $\pm 10 \mathrm{GeV}$ [4]). Moreover, the spectral softening break energy observed in CALET $(11 \pm 4 \mathrm{TeV})$ is consistent with that of DAMPE $\left(13.6_{-4.8}^{+4.1} \mathrm{TeV}\right.$ [4]). These spectral hardening and softening observations are useful to model the mechanisms of cosmic-ray acceleration and propagation in the Galaxy. A recent example can be found in [9]. 


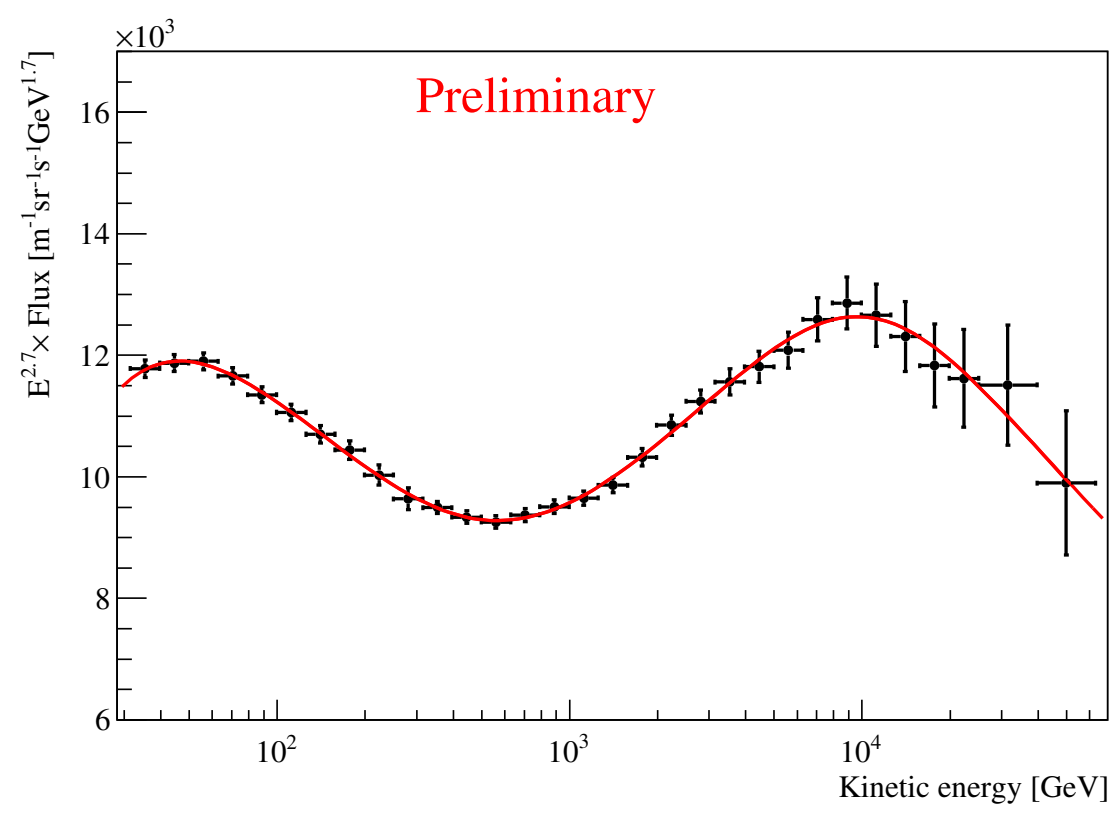

Figure 5: CALET Proton spectrum fitted with the function in Equation 2. The horizontal error bars are representative of the bin width.

\section{Summary}

We have performed an extended measurement of the proton spectrum with CALET data taken from Oct. 2015 to Sep. 2020 increasing the statistics with two additional years of data since we published in 2019. We have confirmed the presence of a spectral hardening around $500 \mathrm{GeV}$ with higher statistics. In addition, we have expanded our energy reach to $60 \mathrm{TeV}$ and we have observed a spectral softening around $10 \mathrm{TeV}$.

\section{References}

[1] O. Adriani et al. (CALET Collaboration), Phys. Rev. Lett. 122, 181102 (2019).

[2] M. Aguilar et al. (AMS Collaboration), Phys. Rev. Lett. 114, 171103 (2015).

[3] Y. S. Yoon et al. (CREAM-III Collaboration), Astrophys. J 839, 5 (2017).

[4] Q. An et al. (DAMPE Collaboration), Science Adv. 5, eaax3793 (2019).

[5] Y. Asaoka et al. (CALET Collaboration), Astropart. Phys. 100, 29 (2018).

[6] O. Adriani et al. (CALET Collaboration), Phys. Rev. Lett. 119, 181101 (2017).

[7] O. Adriani et al. (CALET Collaboration), Phys. Rev. Lett. 120, 261102 (2018). 
[8] K. Kasahara, Proc. 24th Intl. Cosmic Ray Conf., Rome, Italy, ed. by N. Iucci and E. Lamanna (Intl. Union of Pure and Applied Phys. 1, 399 (1995), http://adsabs.harvard.edu/full/1995ICRC....1..399K.

[9] H. Motz et al., PoS ICRC2021, (2021) In press. 


\section{Full Authors List: CALET Collaboration}

O. Adriani ${ }^{1,2}$, Y. Akaike ${ }^{3,4}$, K. Asano ${ }^{5}$, Y. Asaoka ${ }^{5}$, E. Berti ${ }^{1,2}$, G. Bigongiari 6,7 , W. R. Binns ${ }^{8}$, M. Bongi ${ }^{1,2}$, P. Brogi ${ }^{6,7}$, A. Bruno ${ }^{9,10}$, J. H. Buckley ${ }^{8}$, N. Cannady ${ }^{11,12,13}$, G. Castellini ${ }^{14}$, C. Checchia ${ }^{6}$, M. L. Cherry ${ }^{15}$, G. Collazuol ${ }^{16,17}$, K. Ebisawa ${ }^{18}$, A. W. Ficklin ${ }^{15}$, H. Fuke ${ }^{18}$, S. Gonzi ${ }^{1,2}$, T. G. Guzik ${ }^{15}$, T. Hams ${ }^{11}$, K. Hibino ${ }^{19}$, M. Ichimura ${ }^{20}$, K. Ioka $^{21}$, W. Ishizaki ${ }^{5}$, M. H. Israel ${ }^{8}$, K. Kasahara ${ }^{22}$, J. Kataoka ${ }^{23}$, R. Kataoka ${ }^{24}$, Y. Katayose ${ }^{25}$, C. Kato $^{26}$, N. Kawanaka ${ }^{27,28}$, Y. Kawakubo ${ }^{15}$, K. Kobayashi ${ }^{3,4}$, K. Kohri ${ }^{29}$, H. S. Krawczynski ${ }^{8}$, J. F. Krizmanic ${ }^{11,12,13}$, P. Maestro, 6, P. S. Marrocchesi 6,7 , A. M. Messineo ${ }^{30,7}$, J.W. Mitchell ${ }^{12}$, S. Miyake ${ }^{32}$, A. A. Moiseev ${ }^{33,12,13}$, M. Mori ${ }^{34}$, N. Mori ${ }^{2}$, H. M. Motz ${ }^{35}$, K. Munakata ${ }^{26}$, S. Nakahira ${ }^{18}$, J. Nishimura ${ }^{18}$, G. A. de Nolfo ${ }^{9}$, S. Okuno ${ }^{19}$, J. F. Ormes $^{36}$, N. Ospina ${ }^{16,17}$, S. Ozawa ${ }^{37}$, L. Pacini ${ }^{1,14,2}$, P. Papini ${ }^{2}$, B. F. Rauch ${ }^{8}$, S. B. Ricciarini ${ }^{14,2}$, K. Sakai ${ }^{11,12,13 \text {, }}$ T. Sakamoto ${ }^{38}$, M. Sasaki ${ }^{33,12,13}$, Y. Shimizu ${ }^{19}$, A. Shiomi ${ }^{39}$, P. Spillantini ${ }^{1}$, F. Stolzi ${ }^{6,7}$, S. Sugita ${ }^{38}$, A. Sulaj ${ }^{6,7}$, M. Takita ${ }^{5}$, T. Tamura ${ }^{19}$, T. Terasawa ${ }^{40}$, S. Torii ${ }^{3}$, Y. Tsunesada ${ }^{41}$, Y. Uchihori ${ }^{42}$, E. Vannuccini ${ }^{2}$, J. P. Wefel ${ }^{15}$, K. Yamaoka ${ }^{43}$, S. Yanagita ${ }^{44}$, A. Yoshida ${ }^{38}$, K. Yoshida $^{22}$, and W. V. Zober ${ }^{8}$

${ }^{1}$ Department of Physics, University of Florence, Via Sansone, 1, 50019 Sesto, Fiorentino, Italy, ${ }^{2}$ INFN Sezione di Florence, Via Sansone, 1, 50019 Sesto, Fiorentino, Italy, ${ }^{3}$ Waseda Research Institute for Science and Engineering, Waseda University, 17 Kikuicho, Shinjuku, Tokyo 162-0044, Japan, ${ }^{4}$ JEM Utilization Center, Human Spaceflight Technology Directorate, Japan Aerospace Exploration Agency, 2-1-1 Sengen, Tsukuba, Ibaraki 305-8505, Japan, ${ }^{5}$ Institute for Cosmic Ray Research, The University of Tokyo, 5-1-5 Kashiwa-no-Ha, Kashiwa, Chiba 277-8582, Japan, ${ }^{6}$ Department of Physical Sciences, Earth and Environment, University of Siena, via Roma 56, 53100 Siena, Italy, ${ }^{7}$ INFN Sezione di Pisa, Polo Fibonacci, Largo B. Pontecorvo, 3, 56127 Pisa, Italy, ${ }^{8}$ Department of Physics and McDonnell Center for the Space Sciences, Washington University, One Brookings Drive, St. Louis, Missouri 63130-4899, USA, ${ }^{9}$ Heliospheric Physics Laboratory, NASA/GSFC, Greenbelt, Maryland 20771, USA, ${ }^{10}$ Department of Physics, Catholic University of America, Washington, DC 20064, USA, ${ }^{11}$ Center for Space Sciences and Technology, University of Maryland, Baltimore County, 1000 Hilltop Circle, Baltimore, Maryland 21250, USA, ${ }^{12}$ Astroparticle Physics Laboratory, NASA/GSFC, Greenbelt, Maryland 20771, USA, ${ }^{13}$ Center for Research and Exploration in Space Sciences and Technology, NASA/GSFC, Greenbelt, Maryland 20771, USA, ${ }^{14}$ Institute of Applied Physics (IFAC), National Research Council (CNR), Via Madonna del Piano, 10, 50019 Sesto, Fiorentino, Italy, ${ }^{15}$ Department of Physics and Astronomy, Louisiana State University, 202 Nicholson Hall, Baton Rouge, Louisiana 70803, USA, ${ }^{16}$ Department of Physics and Astronomy, University of Padova, Via Marzolo, 8, 35131 Padova, Italy, ${ }^{17}$ INFN Sezione di Padova, Via Marzolo, 8, 35131 Padova, Italy, ${ }^{18}$ Institute of Space and Astronautical Science, Japan Aerospace Exploration Agency, 3-1-1 Yoshinodai, Chuo, Sagamihara, Kanagawa 252-5210, Japan, ${ }^{19}$ Kanagawa University, 3-27-1 Rokkakubashi, Kanagawa, Yokohama, Kanagawa 221-8686, Japan, ${ }^{20}$ Faculty of Science and Technology, Graduate School of Science and Technology,, Hirosaki University, 3, Bunkyo, Hirosaki, Aomori 036-8561, Japan, ${ }^{21}$ Yukawa Institute for Theoretical Physics, Kyoto University, Kitashirakawa Oiwakecho, Sakyo, Kyoto 606-8502, Japan, ${ }^{22}$ Department of Electronic Information Systems, Shibaura Institute of Technology, 307 Fukasaku, Minuma, Saitama 337-8570, Japan, ${ }^{23}$ School of Advanced Science and Engineering, Waseda University, 3-4-1 Okubo, Shinjuku, Tokyo 169-8555, Japan, ${ }^{24}$ National Institute of Polar Research, 10-3, Midori-cho, Tachikawa, Tokyo 190-8518, Japan, ${ }^{25}$ Faculty of Engineering, Division of Intelligent Systems Engineering, Yokohama National University, 79-5 Tokiwadai, Hodogaya, Yokohama 240-8501, Japan, ${ }^{26}$ Faculty of Science, Shinshu University, 3-1-1 Asahi, Matsumoto, Nagano 390-8621, Japan, ${ }^{27}$ Hakubi Center, Kyoto University, Yoshida Honmachi, Sakyo-ku, Kyoto 606-8501, Japan, ${ }^{28}$ Department of Astronomy, Graduate School of Science, Kyoto University, Kitashirakawa Oiwake-cho, Sakyo-ku, Kyoto 6068502, Japan, ${ }^{29}$ Institute of Particle and Nuclear Studies, High Energy Accelerator Research Organization, 1-1 Oho, Tsukuba, Ibaraki 305-0801, Japan, ${ }^{30}$ University of Pisa, Polo Fibonacci, Largo B. Pontecorvo, 3, 56127 Pisa, Italy, ${ }^{31}$ Astroparticle Physics Laboratory, NASA/GSFC, Greenbelt, Maryland 20771, USA, ${ }^{32}$ Department of Electrical and Electronic Systems Engineering, National Institute of Technology, Ibaraki College, 866 Nakane, Hitachinaka, Ibaraki 312-8508, Japan ${ }^{33}$ Department of Astronomy, University of Maryland, College Park, Maryland 20742, USA, ${ }^{34}$ Department of Physical Sciences, College of Science and Engineering, Ritsumeikan University, Shiga 525-8577, Japan, ${ }^{35}$ Faculty of Science and Engineering, Global Center for Science and Engineering, Waseda University, 3-4-1 Okubo, Shinjuku, Tokyo 169-8555, Japan, ${ }^{36}$ Department of Physics and Astronomy, University of Denver, Physics Building, Room 211, 2112 East Wesley Avenue, Denver, Colorado 80208-6900, USA, ${ }^{37}$ Quantum ICT Advanced Development Center, National Institute of Information and Communications Technology, 4-2-1 Nukui-Kitamachi, Koganei, Tokyo 184-8795, Japan, ${ }^{38}$ College of Science and Engineering, Department of Physics and Mathematics, Aoyama Gakuin University, 5-10-1 Fuchinobe, Chuo, Sagamihara, Kanagawa 252-5258, Japan, ${ }^{39}$ College of Industrial Technology, Nihon University, 1-2-1 Izumi, Narashino, Chiba 275-8575, Japan ${ }^{40}$ RIKEN, 2-1 Hirosawa, Wako, Saitama 351-0198, Japan, ${ }^{41}$ Division of Mathematics and Physics, Graduate School of Science, Osaka City University, 3-3-138 Sugimoto, Sumiyoshi, Osaka 558-8585, Japan, ${ }^{42}$ National Institutes for Quantum and Radiation Science and Technology, 4-9-1 Anagawa, Inage, Chiba 263-8555, Japan, ${ }^{43}$ Nagoya University, Furo, Chikusa, Nagoya 464-8601, Japan, ${ }^{44}$ College of Science, Ibaraki University, 2-1-1 Bunkyo, Mito, Ibaraki 310-8512, Japan 